\title{
BMJ Open Critical care work during COVID-19: a qualitative study of staff experiences in the UK
}

\section{Catherine M Montgomery (D) , Sally Humphreys (D) , ${ }^{2}$ Corrienne McCulloch, ${ }^{3}$ Annemarie B Docherty, ${ }^{3,4}$ Steve Sturdy (1) , ${ }^{1,5}$ Natalie Pattison (D) ${ }^{6,7}$}

To cite: Montgomery CM, Humphreys S, McCulloch C, et al. Critical care work during COVID-19: a qualitative study of staff experiences in the UK. BMJ Open 2021;11:e048124. doi:10.1136/ bmjopen-2020-048124

- Prepublication history for this paper is available online. To view these files, please visit the journal online (http://dx.doi. org/10.1136/bmjopen-2020048124).

Received 20 December 2020 Revised 17 March 2021 Accepted 12 April 2021

\section{Check for updates}

(C) Author(s) (or their employer(s)) 2021. Re-use permitted under CC BY. Published by BMJ.

${ }^{1}$ Centre for Biomedicine, Self and Society, University of Edinburgh, Edinburgh, UK ${ }^{2}$ Critical Care and Research \& Development, West Suffolk NHS Foundation Trust, Suffolk, UK

${ }^{3}$ Anaesthetics, Theatres and Critical Care, NHS Lothian, Edinburgh, UK

${ }^{4}$ Centre for Medical Informatics, The Usher Institute, University of Edinburgh, Edinburgh, UK

${ }^{5}$ Science, Technology and Innovation Studies, University of Edinburgh, Edinburgh, UK

${ }^{6}$ School of Health and Social Work, University of Hertfordshire, Hertfordshire, UK

${ }^{7}$ Nursing, East and North Hertfordshire NHS Trust, Stevenage, UK

\section{Correspondence to} Dr Catherine M Montgomery; Catherine.montgomery@ed. ac.uk

\section{ABSTRACT}

Objective To understand National Health Service (NHS) staff experiences of working in critical care during the first wave of the COVID-19 pandemic in the UK.

Design Qualitative study using semistructured telephone interviews and rapid analysis, interpreted using Baehr's sociological lens of 'communities of fate'.

Participants Forty NHS staff working in critical care, including 21 nurses, 10 doctors and advanced critical care practitioners, 4 allied health professionals, 3 operating department practitioners and 2 ward clerks. Participants were interviewed between August and October 2020; we purposefully sought the experiences of trained and experienced critical care staff and those who were redeployed.

Setting Four hospitals in the UK.

Results COVID-19 presented staff with a situation of extreme stress, duress and social emergency, leading to a shared set of experiences which we have characterised as a community of fate. This involved not only fear and dread of working in critical care, but also a collective sense of duty and vocation. Caring for patients and families involved changes to usual ways of working, revolving around: reorganisation of space and personnel, personal protective equipment, lack of evidence for treating COVID-19, inability for families to be physically present, and the trauma of witnessing extreme patient acuity and death on a large scale. The stress and isolation of working in critical care during COVID-19 was mitigated by strong teamwork, camaraderie, pride and fulfilment.

Conclusion COVID-19 has changed working practices in critical care and profoundly affected staff physically, mentally and emotionally. Attention needs to be paid to the social and organisational conditions in which individuals work, addressing both practical resourcing and the interpersonal dynamics of critical care provision.

\section{INTRODUCTION}

COVID-19 has placed unprecedented demands on the UK National Health Service (NHS). Around $8 \%$ of all hospital admissions, over 14000 patients, have been admitted to critical care services with COVID-19 since February 2020. ${ }^{1}{ }^{2}$ Critical care services were rapidly expanded to meet these demands. ${ }^{3}$ Research from China and France into the experiences of healthcare staff demonstrates the enormous

\section{Strengths and limitations of this study}

- This is the first study to provide a sociological analysis of critical care work during the first wave of the COVID-19 pandemic in the UK.

- International studies of staff experience of COVID-19 have focused on individualised mental health outcomes; we use the theoretical concept of 'communities of fate' to add value to existing approaches.

- Our sample included a range of professional groups and explicitly sought to capture the experiences of both experienced and redeployed staff.

- Our sample was limited due to the fact that participants were self-selecting and came from a small number of sites.

- Medical and nursing staff made up the majority of participants in our sample; our findings may overrepresent the experiences of these professional groups thus limiting wider generalisability.

pressure COVID-19 has placed on doctors and nurses, ranging from issues of healthcare service organisation to personal mental health and well-being. ${ }^{45}$ In the UK context, Vindrola-Padros et al report on anxiety and distress caused by limited personal protective equipment (PPE) for healthcare workers, lack of routine testing and unmet training needs for redeployed staff. ${ }^{6}$

Research on healthcare staff during the pandemic has predominantly focused on the psychological impacts of working in critical care during this time. A European-wide study identified high levels of self-reported burnout $(51 \%)$ during the pandemic among intensive care unit (ICU) staff respondents. ${ }^{5}$ We know that burnout, moral injury and moral distress are significant issues in critical care staff, and prior to COVID-19 were already the subject of a call to arms in the international critical care community. ${ }^{7}$ Prevalence in critical care staff ranges from $6 \%$ to $47 \%$, with worse burnout compared with areas such as palliative care, and significant emotional labour associated with working in critical care. ${ }^{8-10}$ 
While much of the research to date has taken a narrowly psychological approach to staff experience, focusing on poor mental health outcomes such as burnout, we aimed to adopt a broader, sociological lens. As Matthewmann and Huppatz note, "As the discipline charged with making sense of contemporary social cohesion and transformation, sociology is well placed to comment on coronavirus and its profound consequences." 11 The sociology of pandemics draws attention to the way in which social institutions-including healthcare systems-change when biological environments change and threaten established ways of living and acting in the world. ${ }^{12}$ Social fragilities and structural deficiencies are laid bare and para-epidemics—of fear, of explanation and moralisation, and of action-proliferate. ${ }^{13}{ }^{14}$ In relation to the impacts of COVID-19 on mental health, members of the Society and Mental Health COVID-19 Expert Group recently argued against the pathologisation of responses to the pandemic and instead called for attention to the social substrates of poor mental health. ${ }^{15}$ This view is echoed by a recent paper by Vera San Juan et al, emphasising the importance of socio-ecological approaches to healthcare worker well-being during the COVID-19 pandemic. ${ }^{16}$ We accordingly aimed to provide an alternative to the discourse of individual psychological responses in healthcare workers by exploring issues including changing work organisation and its impacts, identity and care work, and interpersonal/professional relationships.

We draw on the sociological concept 'community of fate' to illuminate the experiences of frontline staff working in critical care during the first wave of the COVID-19 pandemic in the UK. Analysing the 2003 SARS outbreak in Hong Kong, Baehr describes a 'community of fate' as a particular form of group cohesion arising from a situation of extreme duress (figure 1). ${ }^{17} 18$ Such communities are socially productive; in the face of an existential threat, they mobilise around shared purpose and resources (including organisation and leadership) to instantiate collective action. A key hallmark of such communities is 'a common focus of sustained attention, and an intense feeling of horizontal interconnectedness'. ${ }^{18}$

\section{METHODS}

We conducted qualitative research using semistructured telephone interviews and rapid analysis. ${ }^{19}{ }^{20}$ Qualitative research can contribute to the evidence base of managing COVID-19 by accessing how frontline staff manage their day-to-day work, why particular approaches work or not from the point of view of those implementing them, and what could be done to improve the experience of caring for patients and families in critical care. ${ }^{21}$ Critical care is used as a term throughout to encompass intensive care/ ICUs, intensive therapy units and critical care/critical care units.

\section{Sampling and recruitment}

Using principles of maximum variation sampling, ${ }^{22}$ we recruited 40 frontline staff members working in critical care from four hospitals in the UK, including nurses, medical staff, allied health professionals (AHPs) and ward clerks. The hospitals were all located in urban areas and served populations of between 500000 and 2000000 inhabitants. At the time of the study, hospital A had more than 25 critical care beds, hospitals B and D had between 15 and 20 critical care beds, and hospital $\mathrm{C}$ had fewer than 10 critical care beds. All increased their capacity to deal with the COVID-19 surge in the first wave of the pandemic. We recruited 18 participants from hospital A, 6 from each of hospitals B and C, and 10 from hospital D. The sample was diverse with respect to age, gender and experience working in critical care (table 1). The study was advertised using posters, email and word of mouth. Snowball sampling, which takes advantage of the social networks of identified respondents, provided the research team with an escalating set of participants.

Danger recognition: Awareness of a pressing and immediate danger, which poses a risk to life and common existence

Moral density: Recognition that one's own fate is tie up with others', leading to a sense of social interconnectedness, which may manifest as altruism

Trial: The experience of sustained ordeal

Closure: Literal and metaphorical curtailment of movement; having to stand one's ground, leading to a sense of collective exile and isolation

Material and organisational resources: Resources which help to combat the threat

Axis of convergence: May include a common language or civic pride, through which the sense of community is affirmed

Social ritual: Actions which provide the group with a specific, crisis-related social identity, which separates them from normal life and provides a basis for social cohesion

Figure 1 Key features of communities of fate (adapted from Baehr, 2005). ${ }^{17}$ 


\begin{tabular}{|c|c|c|c|c|}
\hline Profession & Redeployed & Female & Male & Total \\
\hline \multirow{2}{*}{$\begin{array}{l}\text { Advanced critical } \\
\text { care practitioner }\end{array}$} & Yes & - & - & - \\
\hline & No & 1 & - & 1 \\
\hline \multirow[t]{2}{*}{ Dietitian } & Yes & 1 & - & 1 \\
\hline & No & - & - & - \\
\hline \multirow[t]{2}{*}{ Doctor } & Yes & - & 1 & 1 \\
\hline & No & 3 & 5 & 8 \\
\hline \multirow[t]{2}{*}{ Nurse } & Yes & 11 & 2 & 13 \\
\hline & No & 8 & - & 8 \\
\hline \multirow{2}{*}{$\begin{array}{l}\text { Operating } \\
\text { department } \\
\text { practitioner }\end{array}$} & Yes & 2 & 1 & 3 \\
\hline & No & - & - & - \\
\hline \multirow[t]{2}{*}{ Physiotherapist } & Yes & 3 & - & 3 \\
\hline & No & - & - & - \\
\hline \multirow[t]{2}{*}{ Ward clerk } & Yes & 2 & - & 2 \\
\hline & No & - & - & - \\
\hline Total & & 31 & 9 & 40 \\
\hline
\end{tabular}

Interested participants were provided with information about the study, contacted the principal investigator and were subsequently provided with a participant information sheet. Once participants had agreed, verbal informed consent was digitally recorded prior to interview.

\section{Data collection and analysis}

Telephone interviews were conducted between August and October 2020 by CM (sociologist), SH (research nurse/ scholar) and NP (clinical professor of nursing). The research team included the additional expertise of a critical care consultant, professor of sociology and nurse researcher in critical care, with significant combined qualitative research experience. Interviews were digitally recorded and professionally transcribed. Interviews lasted from between approximately 30 and $80 \mathrm{~min}$. Semistructured interviews covered staff's experiences of working in critical care during the first wave of the pandemic (roughly between March and July 2020). Questions related to changes in working practice, interaction with patients, technology for family communication, end of life, learning and training, and personal wellbeing and support.

Data were analysed by team members following the rapid analysis methods proposed by Hamilton ${ }^{19}$ and elaborated by Taylor $e t a l .{ }^{20}$ In the first stage, key issues were noted on a structured summary template describing: participant and data collection details, deductive and inductive headings, quotations and the analyst's reflections. Deductive aspects of the summary template were developed from the research questions. Following an initial testing period, additional, inductively generated subheadings were added. Summarised data were then transferred to a matrix to "streamline the process of noting simultaneously and systematically similarities, differences and trends in responses across groups of informants'. ${ }^{23}$
Transparent team review and discussion across all transcripts took place to enhance confirmability, trustworthiness, dependability and credibility. ${ }^{24}$ Early findings were discussed within the team and subsequently interpreted using the sociological lens of 'communities of fate'. ${ }^{17}$

\section{Patient and public involvement}

Patients and/or the public were not involved in the design, conduct, reporting or dissemination of this study.

\section{RESULTS}

COVID-19 presented staff in critical care with a rapidly changing situation and guidance; staff found themselves working in a state of constant flux. Participants' accounts reflected changes every shift, with one person saying every day felt like the first day in a new job. Below, we describe the features of working in this extreme pandemic context in relation to the seven features of a community of fate, highlighting not just changes in working practice but the social corollaries of these changes. Illustrations from the data are given in figures $2-5$.

\section{Danger recognition: fear and dread of COVID-19}

Many staff members commented on the anxiety they felt in anticipation of working in critical care during COVID-19. During the early stage of the pandemic, these anxieties were heightened by media reports of overwhelmed hospitals in Italy and exhausted healthcare workers in China. Staff were also acutely aware of their personal risk of catching COVID-19 and taking this home to their families. Particularly affected were those from ethnic minorities and those with at-risk and shielding family members. One black African nurse living in a multigenerational household described the impact the death of a fellow nurse from COVID-19 had had on her, and how her sister, also a nurse, had also fallen ill with COVID19. Such experiences heightened the anxiety of working in critical care; they also entailed considerable emotional labour-something described by several respondents when talking about how they had sought to reassure their partners and children that they were safe at work. A number of participants had experienced the death of colleagues, which was deeply affecting.

\section{Moral density: purpose and duty}

In spite of-indeed because of-this existential threat, many staff members spoke of a strong sense of duty in relation to working during COVID-19. Recognition of the danger the pandemic posed to the population as a whole was a powerful motivator, prompting several redeployed staff members in the sample to proactively volunteer. Shared professional commitment was also a powerful factor, with some expressing that this was simply their job, and others that it was what they had been trained to do. This collective vocation to provide care and be present created a common sense of purpose, which cut across professions and hierarchies. 


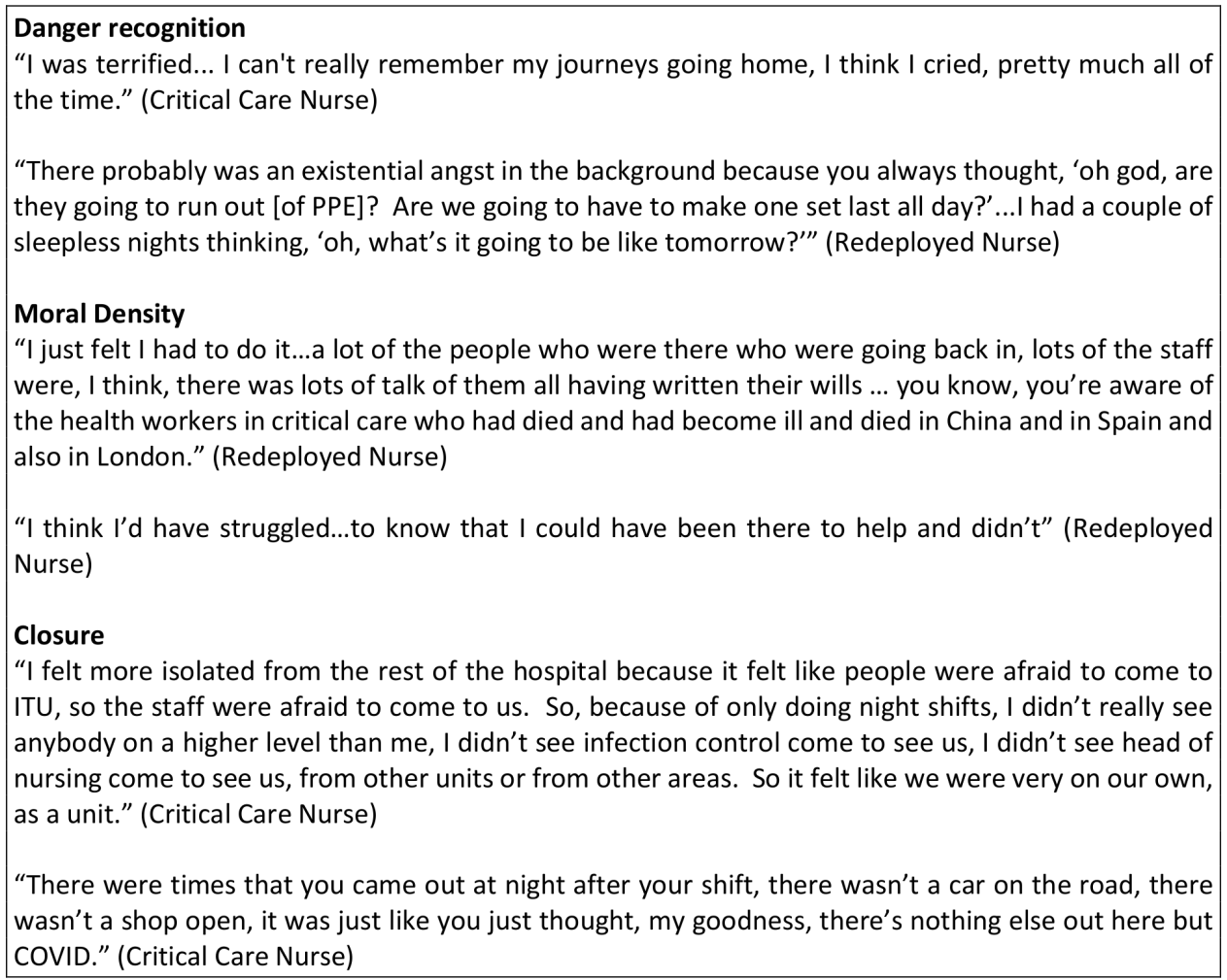

Figure 2 Data extracts illustrating critical care as a community of fate during COVID-19. ITU, intensive therapy unit; PPE, personal protective equipment.

\section{Trial: ordeals in critical care}

Working in COVID-19 critical care was extremely challenging. The main ordeals that staff described were as follows (see also figures 3 and 4):

\section{Dislocation}

Setting up COVID-19 critical care facilities often involved converting clinical areas, including wards and theatre recovery areas, to new purposes and assimilating redeployed staff into newly assembled critical care teams. Adapting to these new circumstances proved challenging, with staff reporting difficulties locating equipment and supplies, or identifying who was in charge. Lack of familiarity with other team members was exacerbated by PPE, which rendered identification and recognition difficult. Redeployed staff without previous critical care experience faced particularly acute challenges adapting to unfamiliar language and processes, with some saying they felt ill equipped to deal with even basic tasks such as how to record observations or wash patients. This was particularly so for the operating department practitioners (ODPs) in our sample.

\section{Responsibility}

Staff described a rapid acceleration in levels of responsibility. This included managing the dual tasks of caring for critically ill patients while also training non-critical care staff, which created additional cognitive and emotional demands: some senior nursing staff reported not taking a break for 6 or 7 hours due to anxiety over leaving inexperienced staff. These demands were magnified for staff from smaller units with a smaller pool of experienced staff to draw on for redeployment into critical care. Extreme stress was reported by senior nursing staff trying to maintain adequate staffing levels in these contexts. The sheer number of patients also exacerbated the burdens of responsibility. The decision to abandon existing guidance on minimum staff:patient ratios $^{25}$ was perceived to be unsafe by some nursing staff, and led to a loss of confidence, even for some experienced nurses. Some reported suffering from extreme anxiety and a sense of loss of control when attempting to look after patients safely and with dignity, while nurses in several units expressed sadness at their inability to provide as much care as usual in terms of washing, turning and personal care.

\section{Caring for patients}

Staff commented repeatedly on the physical and emotional intensity of caring for critically ill patients with COVID-19, and their acute awareness of how frightening it was for patients. While the majority of staff felt safe and protected by their PPE, it nonetheless made caring for patients difficult due to loss of manual dexterity, numbing of the senses, loss of visual and audio cues, heat, weight, dehydration, facial pain and the fact that everyone looked the same. This also disrupted professional interaction: staff were sometimes unable to recognise who had the expertise to respond to a given request for help, and it hindered tasks that depended on coordinated teamwork such as proning or turning patients. 


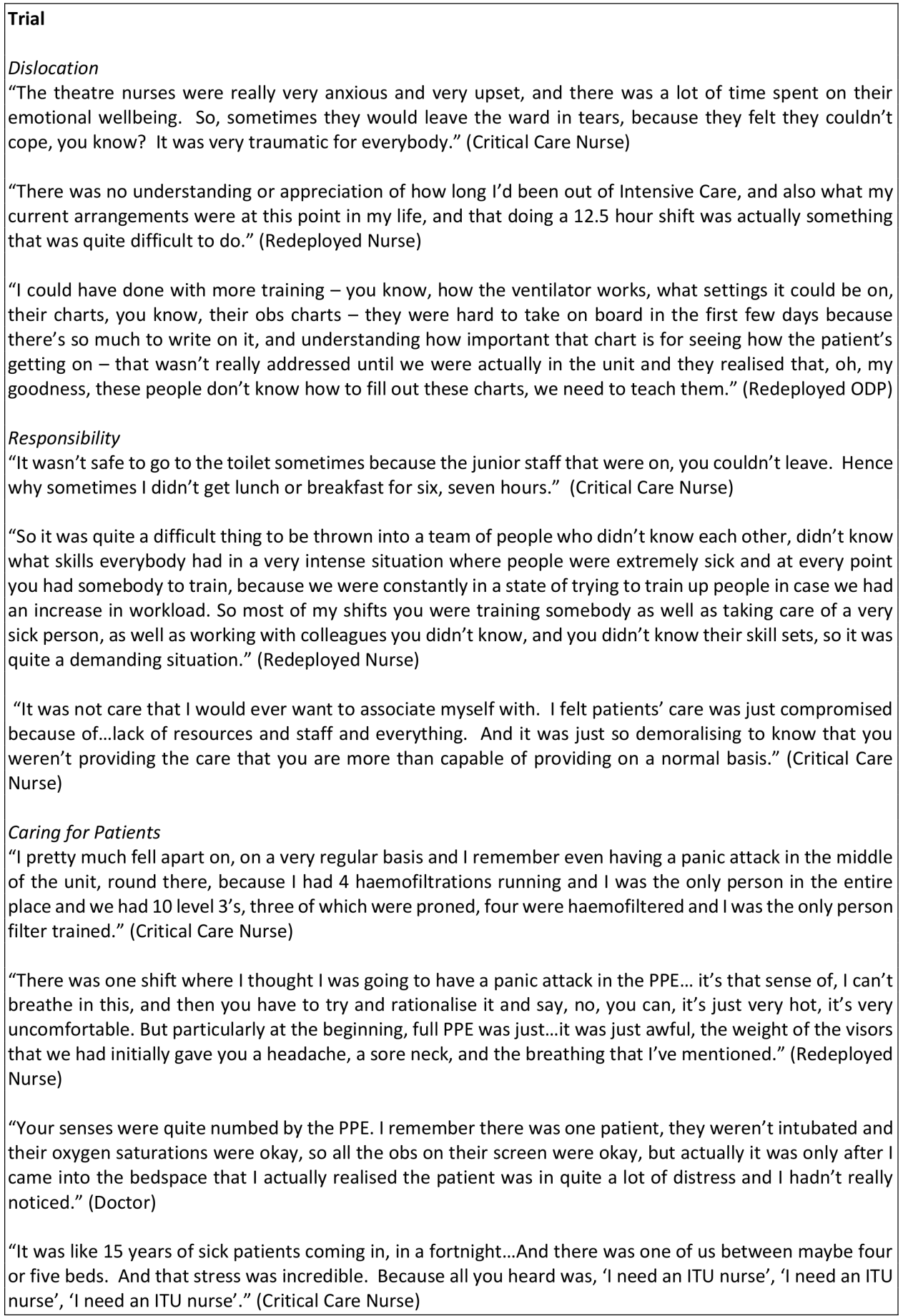

Figure 3 Data extracts illustrating critical care as a community of fate during COVID-19. ITU, intensive therapy unit; ODP, operating department practitioner; PPE, personal protective equipment.

The lack of an evidence base for treating COVID-19 also led to fundamental uncertainty about what care to provide. Medical and nursing staff alike commented on the simultaneous information vacuum and information deluge, compounded by the lack of a central, controlled source for information about clinical practice, with much communicated by word of mouth. AHPs described new challenges associated with 'caring at a distance', when direct access to patients was either not possible or limited. For example, one dietitian spoke of how relying on verbal reports from nursing staff via telephone rather than seeing the patient and their charts themselves made it difficult to assess nutritional status and ensure appropriate supplies.

\section{End of life}

While many participants were used to caring for dying patients, COVID-19 brought new difficulties. With families unable to visit, staff's emotional relationship with patients was intensified, with many staff members saying 


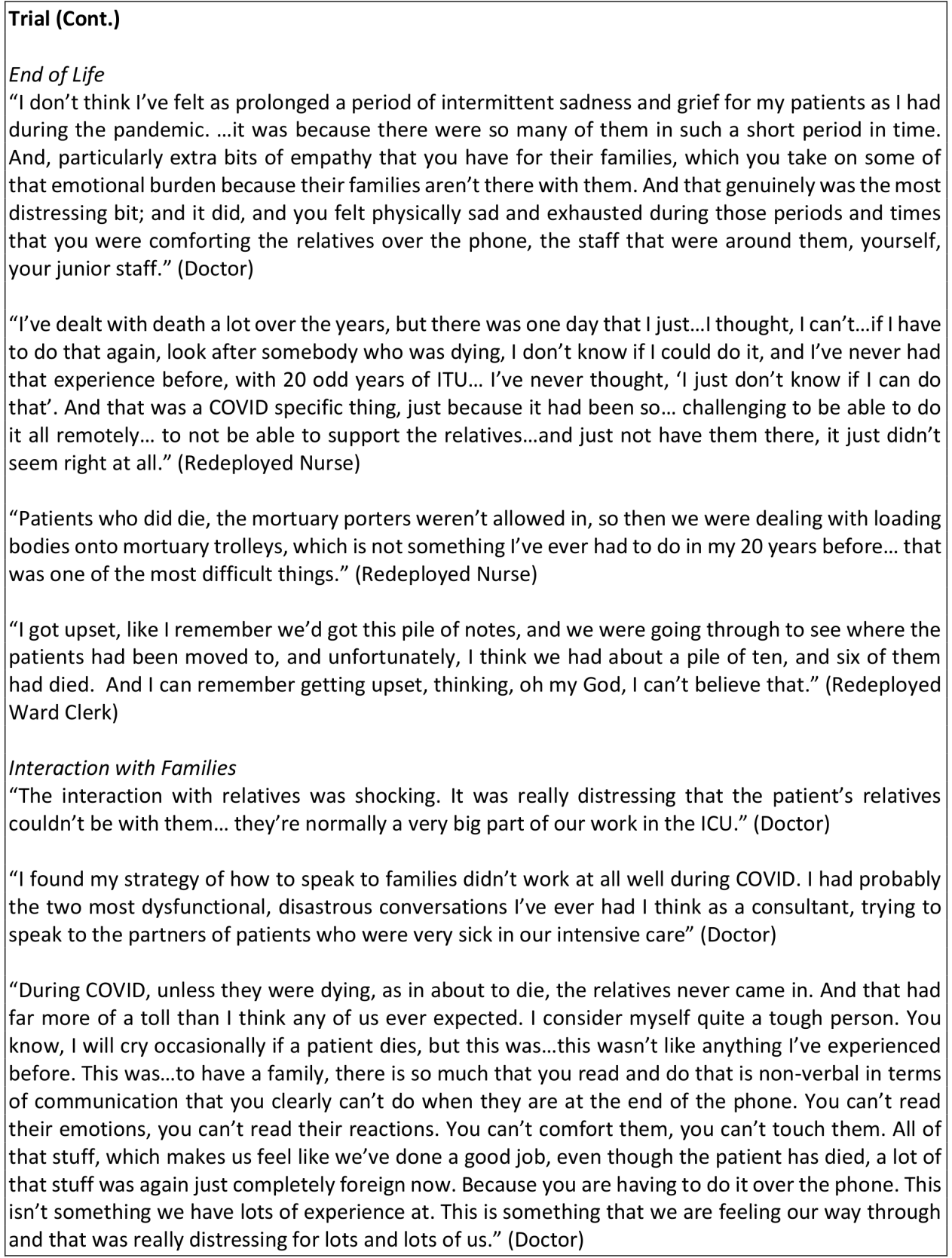

Figure 4 Data extracts illustrating critical care as a community of fate during COVID-19. ICU, intensive care unit; ITU, intensive therapy unit.

they felt they had to take on the family's role of 'being there' for the patient. All the staff members we interviewed expressed deep sadness at witnessing the deaths of patients with COVID-19 who were not allowed to have a family member present in person. Staff who sat with patients at the end of life often found it heartbreaking to be party to this very intimate moment between a patient and their family, for example, while holding a telephone to the patient's ear. Staff reported that the decision to allow family members in again towards the end of life had made a huge difference and 'humanised the process again'.

Staff also encountered new challenges after a patient had died. Some nurses reported that protocols regarding what to do after a patient had died from COVID-19 were not clear at the start, for example, around last offices, infection control and what to do with patient belongings. One experienced nurse described how upsetting it was to be tasked with moving people who had died into body bags and onto trolleys for the morgue.

The severity of illness and high death rate in COVID-19 critical care, while difficult for all staff, was particularly hard for redeployed theatre and recovery staff, whose work usually involves patients who improve. The ODPs we spoke to reported having no training or experience in communicating with families at end of life. Ward clerks were also affected by the sheer numbers of deaths and caring for these patients' families. As the first port of call 


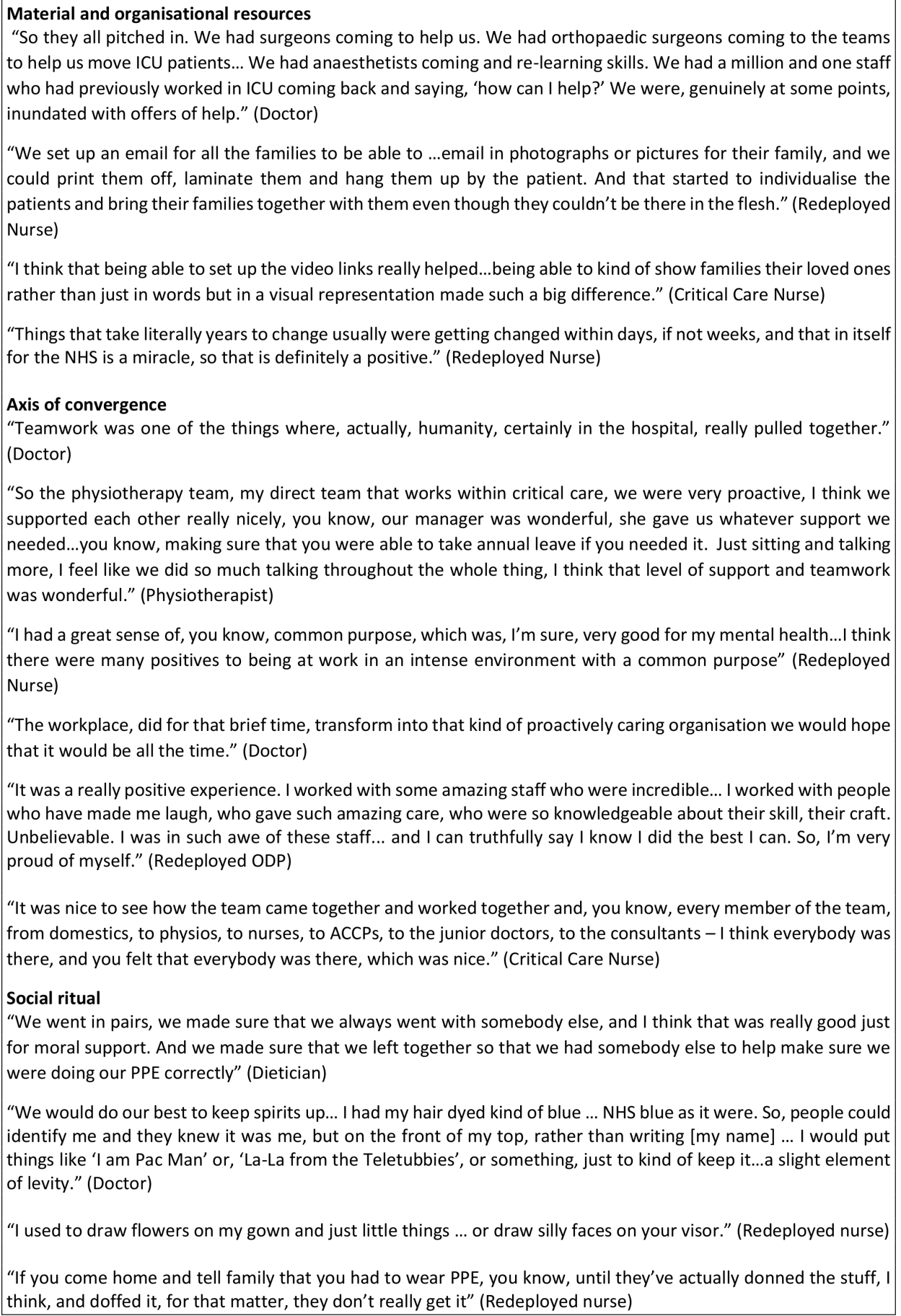

Figure 5 Data extracts illustrating critical care as a community of fate during COVID-19. ACCPs, advanced critical care practitioners; ICU, intensive care unit; NHS, National Health Service; ODP, operating department practitioner; PPE, personal protective equipment.

for families phoning up, this work could be very emotionally intense; as one ward clerk observed, 'sometimes you could just listen to them, even though you couldn't help them'.

\section{Interaction with families}

Caring for families is a large part of critical care work, ${ }^{26} 27$ and particularly important at the end of a patient's life. ${ }^{28} 29$ With visitors excluded from patients' bedsides due to COVID-19, staff experienced additional demands to keep families informed while navigating the constraints of communicating 'virtually' using digital technologies/telephone. Staff spoke of the peculiar difficulties of avoiding unwarranted optimism or pessimism when families were unable to witness for themselves how a patient might be progressing or deteriorating. The need to communicate at a distance also made it particularly challenging for staff to break bad news. Most staff felt unprepared to have these conversations, and consultants, 
in particular, reported having some of the most upsetting conversations of their careers.

The emotional strain of facing these trials, combined with the sense of isolation, was often severe. Some nursing staff reported experiencing acute fear, stress, anxiety, exhaustion and burnout, particularly in smaller units. Staff spoke of crying on the way to work, breaking down in tears on shift and crying after leaving work. Fear for the consequences of what they perceived to be inadequate staffing levels, inexperienced staff and a high volume of critically ill patients left some nursing staff feeling 'broken'. Across the sample, staff reported a range of negative impacts, such as sleep disturbance, panic attacks, weight loss/gain, and feelings of guilt, grief, anger, sadness and dread. Some accessed professional mental health services, either within the Trust, through their general practitioner or privately.

\section{Closure: isolation and the 'COVID-19 bubble'}

With COVID-19 critical care facilities physically and socially isolated from other parts of the hospital for infection control purposes, and many staff members removed or redeployed from their usual workplaces and colleagues, work in what some staff referred to as the 'COVID-19 bubble' could feel like a collective exile. Several participants voiced disappointment that COVID-19 seemed to be treated as 'a problem for critical care' rather than the hospital at large, such was their sense of isolation. This was magnified for staff working night shifts, who commented on feeling forgotten, having less food and drink available, fewer redeployed staff to support them and less visibility of senior managers.

\section{Material and organisational resources: learning and creativity}

By contrast, staff also took pride in the many ways they had managed to adapt to the challenges posed by new working arrangements. Staff experienced a steep learning curve: consultants described the challenges of treating patients in the absence of an evidence base, while nurses spoke of learning to manage a large number of patients who were considerably sicker than usual and whose condition could deteriorate quickly. Both groups spoke of the quick, self-directed learning needed to stay abreast of rapidly changing treatment protocols. While some felt that 'nothing prepares you to work in a pandemic', those with experience of working in previous infectious disease outbreaks such as SARS or H1N1, as well as those involved in protocol development and training for emergency situations, felt better prepared.

Rapid learning across units was helped by the relative homogeneity of the cohort of patients with COVID-19. The following training was specifically mentioned as helpful: locally run, structured competency and skills training; the FutureLearn COVID-19 Critical Care course; and the frequent Intensive Care Society webinars. For nursing staff, much of the training occurred on the job; those who had been redeployed particularly valued the opportunity to shadow staff and/or to have a more experienced buddy. Staff across nursing and medical teams identified platforms like WhatsApp as a crucial means of sharing information and updates, moving away from traditional modes of communication.

There was widespread praise for the speed with which new systems had been put in place and change effected in the NHS. A particular success was the use of tablets and mobile phones to connect families and patients via synchronous/asynchronous video-conferencing. Staff observed that the more frequently they were able to connect with families, the easier this virtual relationship became, the more patients were individuated, and the more satisfactory the caring relationship.

\section{Axis of convergence: teamwork}

Almost everyone we interviewed articulated positive aspects of their experience during the pandemic. Foremost among these was the sense of teamwork and camaraderie that had developed as staff pulled together. Many felt proud to have been part of the pandemic response, and spoke of the satisfaction of being part of something and working for a common purpose, while the influx of redeployed staff was often felt to be a source of both moral and practical support. Newly qualified and experienced nurses alike said that working during COVID-19 reaffirmed the values that had taken them into the profession in the first place.

Teamwork, and the mutual support it provided, also characterised some of the measures that proved most effective in meeting the challenges of COVID-19. Daily team huddles were observed to be useful for identifying and trouble-shooting local issues, while shared conversations within the team about difficult shifts and patients who had died was helpful in coping with emotionally difficult experiences. As a result, various staff said they had gained improved clinical, operational and management skills, increased resilience, confidence and self-esteem.

\section{Social rituals: donning and doffing}

In a setting that depends so heavily on teamwork, it would be futile to try and draw any hard-and-fast distinction between routine and ritual: both serve at once to provide reassurance and to affirm a common identity in the face of disorder and danger. In the context of COVID-19, however, some routines acquired special significance. The donning and doffing of PPE was one such. Staff spoke of the benefits of going into critical care in pairs to check both on PPE fit and each other's well-being. They also found creative ways to decorate their PPE, turning it from a faceless signifier of risk into an expression of individuality. In so doing, they transfigured the fear of entering critical care into a moment of human solidarity and interconnectedness.

\section{DISCUSSION}

Using the sociological concept of 'community of fate', our analysis shows how working practice in critical care 
changed during the first wave of the COVID-19 pandemic in the UK, and how staff mobilised their collective resources to provide care to patients. In Baehr's Weberian use of the term, 'community of fate' does not imply fatalism. On the contrary, it denotes the condition of purposeful collective action that may be attained by a group of people facing a common crisis. Employing the concept to analyse our interviewees' testimony not only helps explain staff experience in the face of extremity, but highlights the crucial role of solidarity and teamwork in achieving a functioning COVID-19 critical care system.

That collective achievement should not blind us to the anguish that many participants endured and its lasting damage: several staff expressed a deep reluctance to return to critical care to tackle a second wave, something highlighted in other qualitative studies of frontline staff experience ${ }^{30}$ Nor should we assume that the communities of fate that coalesced in response to the first wave will survive as the pandemic becomes increasingly protracted and challenges recur. As Baehr notes "Where all hope is gone, resources spent, and action deemed hopeless, communities of fate are impossible." ${ }^{" 18}$ It therefore behoves us to ensure that resources are available and the conditions for good care and staff well-being are optimised. While some have suggested this should focus on individual well-being initiatives, including mindfulness and other coping strategies, ${ }^{31}$ our analysis underscores the importance of structural resilience in critical care and attending to the conditions under which teams can prosper.

Our study is not alone in emphasising the importance of taking healthcare workers' experiences into account during the COVID-19 pandemic. Indeed, our findings echo and amplify many of those from the Rapid Research, Evaluation and Appraisal Lab Study of the perceptions and experiences of healthcare workers during COVID$19{ }^{61632}$ While the latter drew on a sample consisting primarily of doctors, our study adds the voices of nurses and other professionals to the evidence base, as well as extending its geographical reach beyond London to other parts of the UK. By drawing on sociological theory to interpret our data, we provide an alternative lens through which to understand social cohesion and transformation in critical care during pandemic times.

Some limitations to our study apply, namely the selfselecting sample, the small number of sites from which we recruited and the small number of AHPs. As such, our findings may over-represent the experiences of nursing and medical staff, and further research should consider a broader range of experiences from across the professions. While we recruited from a range of critical care settings across the UK, these were all located in urban areas and staff working in rural areas may have different experiences. Nonetheless, we believe the concept of 'communities of fate' is likely to have broad theoretical generalisability. Finally, the rapid analytical methods we used were designed to structure the analysis thematically, and were not explicitly oriented to exploring differences by demographic variables, such as gender and ethnicity.
Studies of staff experience during COVID-19 have shown how these variables shape experiences; ${ }^{32}{ }^{33}$ we acknowledge the importance of recognising the "stratified forms of risks and vulnerabilities facing diverse groups of healthcare workers both within and across health systems'. ${ }^{34}$

A key strength of this study is its in-depth focus on critical care during the first wave of the COVID-19 pandemic in the UK. Our data have alerted us to a range of specific measures that might be implemented at the local level to help critical care staff contend with the challenges posed by COVID-19, and we have made our recommendations available and accessible. ${ }^{35}$ They join a growing body of guidance and resources aimed at helping staff maintain mental health, well-being and resilience through the pandemic. ${ }^{36}{ }^{37}$ As has been noted elsewhere, ${ }^{16}$ with some notable exceptions, ${ }^{38}$ these resources are overwhelmingly oriented towards supporting individuals. Yet as Rose $e t$ $a l^{15}$ argue, individualised psychology-based interventions will be ineffective unless the social preconditions for wellbeing are in place. Our own findings strongly support that view. While personalised support is to be welcomed, attention also needs to be paid to the social and organisational conditions in which individuals work.

Our research shows the importance, on the one hand, of building and facilitating teamwork within and across critical care; and on the other hand, of addressing the sense of isolation that critical care staff felt from other parts of the healthcare system. Resourcing is one aspect of this: our data attest to the need to address anxieties around practical issues such as staffing levels and PPE availability. But equally important is how institutions and national bodies develop transparent plans to deal with COVID-19 and meaningfully engage with frontline staff. Responsibility is key, and time, energy and resource must underpin the professional duty of care to healthcare colleagues in order to comprehensively manage surge situations like the COVID-19 pandemic.

\section{CONCLUSION}

To the best of our knowledge, this is the first sociological analysis of healthcare staff's experiences of working in critical care during the first wave of the COVID-19 pandemic in the UK. Our findings provide timely insight into the challenges of critical care work during the first wave of COVID-19 and suggest the importance of moving beyond an individualised understanding of staff well-being to consider the social and organisational factors at stake.

Twitter Catherine M Montgomery @Cath_Montgomery, Sally Humphreys @ Research2Note, Annemarie B Docherty @abdocherty79 and Natalie Pattison @ drnatpat

Acknowledgements We would like to thank all the NHS staff who took part in this study for giving up their time and sharing their experiences with us for this research.

Contributors CMM and ABD conceived the study, and all authors contributed to the study design. CMM, SH and NP collected the data. CMM, SH, CMc, SS and NP analysed the data and all authors contributed to data interpretation. CMM, SS and 
NP wrote the first draft of the article and all authors contributed to subsequent revisions. All authors had full access to the data.

Funding This research was funded by Medical Research Scotland through a COVID-19 Research Grant (CVG-1739-2020) and supported in part by the Wellcome Trust (209519/Z/17/Z)

Disclaimer The funders of the study had no role in study design, data collection, data analysis, data interpretation or writing of the manuscript.

Competing interests None declared.

Patient consent for publication Not required.

Ethics approval Ethical approval was granted by the University of Edinburgh School of Social and Political Science Research Ethics Committee; HRA approval (20/HRA/3270) was also obtained.

Provenance and peer review Not commissioned; externally peer reviewed.

Data availability statement The datasets used and analysed during the current study are available from the corresponding author on reasonable request.

Open access This is an open access article distributed in accordance with the Creative Commons Attribution 4.0 Unported (CC BY 4.0) license, which permits others to copy, redistribute, remix, transform and build upon this work for any purpose, provided the original work is properly cited, a link to the licence is given, and indication of whether changes were made. See: https://creativecommons.org/ licenses/by/4.0/.

\section{ORCID iDs}

Catherine M Montgomery http://orcid.org/0000-0002-5829-6137

Sally Humphreys http://orcid.org/0000-0002-4397-6404

Steve Sturdy http://orcid.org/0000-0002-3273-1727

Natalie Pattison http://orcid.org/0000-0002-6771-8733

\section{REFERENCES}

1 ICNARC. ICNARC report on COVID-19 in critical care: England, Wales and Northern Ireland, 2020.

2 Data.gov.uk. Coronavirus (COVID-19) in the UK. Patients admitted to hospital 2020. Available: https://coronavirus.data.gov.uk/details/ healthcare [Accessed 9 Nov 2020].

3 NAO. Investigation into how government increased the number of ventilators available to the NHS in response to COVID-19, 2020. Available: https://www.nao.org.uk/report/increasing-ventilatorcapacity-in-response-to-covid-19/

4 Liu Q, Luo D, Haase JE, et al. The experiences of health-care providers during the COVID-19 crisis in China: a qualitative study. Lancet Glob Health 2020;8:e790-8.

5 Azoulay E, De Waele J, Ferrer R, et al. Symptoms of burnout in intensive care unit specialists facing the COVID-19 outbreak. Ann Intensive Care 2020;10:110.

6 Vindrola-Padros C, Andrews L, Dowrick A, et al. Perceptions and experiences of healthcare workers during the COVID-19 pandemic in the UK. BMJ Open 2020;10:e040503.

7 Moss M, Good VS, Gozal D, et al. An official critical care societies collaborative statement: Burnout syndrome in critical care healthcare professionals: a call for action. Crit Care Med 2016;44:1414-21.

8 Chuang $\mathrm{CH}$, Tseng PC, Lin CY. Burnout in the intensive care unit professionals: a systematic review. Medicine 2016;95:e5629.

9 Martins Pereira S, Teixeira CM, Carvalho AS, et al. Compared to palliative care, working in intensive care more than doubles the chances of burnout: results from a nationwide comparative study. PLoS One 2016;11:e0162340.

10 Sorensen R, ledema R. Emotional labour: clinicians' attitudes to death and dying. J Health Organ Manag 2009;23:5-22.

11 Matthewman S, Huppatz K. A sociology of Covid-19. J Sociol 2020;56:675-83

12 Dingwall R, Hoffman LM, Staniland K. Introduction: why a Sociology of Pandemics? Sociol Health IIIn 2013;35:167-73.

13 Pickersgill M. Pandemic sociology. Engag Sci Technol Soc 2020;6:347-50.

14 Strong P. Epidemic psychology: a model. Sociol Health IIIn 1990;12:249-59.

15 Rose N, Manning N, Bentall R, et al. The social underpinnings of mental distress in the time of COVID-19 - time for urgent action. Wellcome Open Res 2020;5:166.
16 Vera San Juan N, Aceituno D, Djellouli N, et al. Mental health and well-being of healthcare workers during the COVID-19 pandemic in the UK: contrasting guidelines with experiences in practice. BJPsych Open 2021;7:e15.

17 Baehr P. Social extremity, communities of fate, and the sociology of SARS. Eur J Sociol 2005;46:179-211.

18 Baehr P. Communities of fate and the SARS emergency in Hong Kong. Caesarism, charisma and fate historical sources and modern resonances in the work of max Weber. New Brunswick, USA and London, UK: Transaction Publishers, 2008.

19 Hamilton A. Qualitative methods in rapid turn-around health services research. VA HSR\&D National Cyberseminar Series: Spotlight on Women's Health, 2013December. Available: https://www.hsrd. research.va.gov/for_researchers/cyber_seminars/archives/video_ archive.cfm?SessionID=780

20 Taylor B, Henshall C, Kenyon S, et al. Can rapid approaches to qualitative analysis deliver timely, valid findings to clinical leaders? a mixed methods study comparing rapid and thematic analysis. BMJ Open 2018;8:e019993.

21 Green J, Thorogood N. Qualitative methods for health research. 2nd edn. London: Sage, 2009.

22 Patton MQ. Qualitative evaluation and research methods. 2nd edn. Newbury Park, California: Sage, 1990.

23 Averill JB. Matrix analysis as a complementary analytic strategy in qualitative inquiry. Qual Health Res 2002;12:855-66.

24 Denzin NK, Lincoln YS. The SAGE Handbook of qualitative research. Thousand Oaks: Sage, 2011.

25 NHS England. Joint statement on developing immediate critical care nursing capacity: 25 March 2020, 2020. Available: https:// www.england.nhs.uk/coronavirus/publication/joint-statement-ondeveloping-immediate-critical-care-nursing-capacity/

26 Davidson JE. Family-centered care: meeting the needs of patients' families and helping families adapt to critical illness. Crit Care Nurse 2009;29:28-34. quiz 5.

27 Kynoch K, Cabilan CJ, McArdle A. Experiences and needs of families with a relative admitted to an adult intensive care unit: a qualitative systematic review protocol. JBI Database System Rev Implement Rep 2016;14:83-90.

28 Faculty of Intensive Care Medicine. Care at the end of life: a guide to best practice, discussions and decision-making in and around critical care. London: FICM, 2019

29 Pattison N, Carr SM, Turnock C, et al. 'Viewing in slow motion': patients', families', nurses' and doctors' perspectives on end-of-life care in critical care. J Clin Nurs 2013;22:1442-54.

30 Bennett P, Noble S, Johnston S, et al. COVID-19 Confessions: a qualitative exploration of healthcare workers experiences of working with COVID-19. BMJ Open 2020;10:e043949.

31 Aughterson H, McKinlay AR, Fancourt D, et al. Psychosocial impact on frontline health and social care professionals in the UK during the COVID-19 pandemic: a qualitative interview study. BMJ Open 2021;11:e047353.

32 Hoernke K, Djellouli N, Andrews L, et al. Frontline healthcare workers' experiences with personal protective equipment during the COVID-19 pandemic in the UK: a rapid qualitative appraisal. BMJ Open 2021;11:e046199.

33 Regenold N, Vindrola-Padros C. Gender Matters: A Gender Analysis of Healthcare Workers' Experiences during the First COVID-19 Pandemic Peak in England. Soc Sci 2021;10:43.

34 Smith $\mathrm{C}$. The structural vulnerability of healthcare workers during COVID-19: observations on the social context of risk and the equitable distribution of resources. Soc Sci Med 2020;258:113119.

35 Montgomery C, Docherty A, Humphreys S, et al. The clap study: summary of findings: University of Edinburgh, 2020. Available: https://www.ed.ac.uk/usher/biomedicine-self-society/centre-news/ the-clap-study-summary-of-findings

36 NHS England. NHS strengthens mental health support for staff, 2020. Available: https://www.england.nhs.uk/2020/10/strengtheningmental-health-support-for-staff/

37 NHS Education for Scotland. Coping with Coronavirus : advice for ICU health care staff, 2020. Available: https://learn.nes.nhs.scot/ 28082/psychosocial-mental-health-and-wellbeing-support/takingcare-of-myself/coping-with-coronavirus-advice-for-icu-health-carestaff

38 Intensive Care Society. Intensive care Society: wellbeing and psychological resource library, 2020. Available: https://www.ics.ac. uk/ICS/Education/Wellbeing/ICS/Wellbeing.aspx 